\title{
CHAPTER SIX
}

\section{The history and application of Additive Manufacturing for Design Personalisation}

\author{
by Guy Bingham
}

\section{Introduction}

Public awareness of Additive Manufacturing (AM) technologies, more popularly known as 3D Printing, has escalated dramatically during the last five years and it is now commonplace to see news and stories about it in the mainstream media. However, the public's appreciation of the technical limitations and implications of the technology is still quite limited - the popular perception of 'anything can be printed' is somewhat misguided. Nevertheless, this perception is part of the allure of $A M$ and its almost magical realisation of 3D objects has ignited interest and captured the public's imagination. The increasing interest has been further solidified by the apparent accessibility of the technology and the affordability and capability of desktop 3D printing. A growing number of home, desktop systems can be purchase for a few hundred pounds and these are helping provide a new creative outlet for the emergent population of home-based 'hackers, makers and tinkerers' (MakerSpace 2015).

The accessibility and the apparent capability characteristic of AM is in stark contrast to traditional manufacturing techniques, especially those developed for mass-production, such as injection moulding. Their costs and need for significant infrastructure make these industrial processes completely inaccessible to the general public. AM technologies are fundamentally different from such processes in practically every aspect. They are digital, flexible and, because they are now available as desktop systems, they are affordable. However, there are significant differences between AM technologies that can be 'industrialised' and the increasingly popular desktop 3D printers that have captured so much public interest. 
What is not so apparent to the general public, and may never need to be, is the significant volume of research and development work that led to the current era of desktop 3D printing. Research in the area of AM is of long standing and can come as a surprise that the initial development work in the field started in the early 1980 's, with the first working Stereolithography system being produced in 1984 (Hull 1986). This was a very exciting period for the technology and in the decade after Hull's Stereolithography process came alternatives like Laser Sintering (Deckard and Beaman 1989), Fused Deposition Modelling (Crump 1992) and a host of different technologies that all allowed the digital, additive fabrication of 3D geometries. While the development of these new systems was quite quick, it initially lacked an application in production and led to their use as prototyping systems, which ultimately led to them being identified under the catch-all phrase 'Rapid Prototyping' (RP).

The term 'Rapid Prototyping' is somewhat misleading and anyone with first-hand experience of using the technology would struggle to describe it as anything near rapid. The term was developed in the 1980 's by 3D Systems - the US-based company and the leader in consumer and industrial 3D printing and manufacturing - to capture the capability of the technology when directly comparing it to the alternative and established techniques of model making being used for the production of prototypes. Model making is a high level craft but the timescale for realising intricate and detailed 3D prototypes using these skills can be measured in weeks and months. The development of RP systems reduces this to hours and days, establishing RP as the most effective process for efficient and accurate realisation of 3D physical prototypes.

Research surrounding RP has continued and concentrated on improving the accuracy, reliability and efficiency of the various technologies available and on developing effective build materials. During the mid-1990's advances in Computer Aided Design (CAD) software and the general increase in desktop computing power allowed a new avenue of research to develop, specifically investigating the design potential or 'design freedom' of additive techniques (Campbell 1994, Burns 1995). The flexibility of RP was already appreciated by the design community but during this period it was realised that additive techniques could facilitate a new level of geometric freedom unimaginable 
from the perspective of conventional manufacturing techniques. While much has been reported on the design freedom of AM and its capability to create 'impossible' geometries, it is important to note that by definition, nothing that can be achieved is actually impossible and it is more meaningful to say that AM has the capability to create highly complex geometries that are simply impractical by any other means. Given enough time, patience and skill, many supposedly 'impossible' geometries could be made by hand but the true advantage of AM lies in its ability to realise impractical forms, more efficiently and repeatably, without needing great fabrication skill. However, skill has not been completely removed from the process of generating such complex and impractical geometries, rather it has been shifted from one stage of the fabrication process to another. Digital manufacturing techniques need digital inputs to generate physical outputs and it is here that high levels of skill are still required, in the operation of $3 D$ CAD and the generation of complex 3D manufacturing data. The need for this high level of skill with CAD is often highlighted as one of the barriers preventing the wider-scale adoption of AM techniques which remains a significant area of research activity today (Hopkinson et al 2005). However much hyped, the design freedom and its research surrounding AM have helped develop some of the core philosophies of the technology and provided important direction for its future research, development and application.

During the early 2000's, the dramatically improving accuracy, reliability, efficiency and build materials, in conjunction with the realised design freedom, led to the desire to elevate the technology from a prototyping platform into a manufacturing process. Originally named 'Rapid Manufacturing', denoting a natural progression from Rapid Prototyping, this description was quickly replaced with 'Additive Manufacturing', pioneered by Neil Hopkinson and Philip Dickens (Hopkinson and Dickens 2003). They proposed that the technology would eventually mature and directly compete with the established mass-manufacturing processes in terms of accuracy and the quality of products/components. They also highlighted that removing all tooling (moulds, jigs and fixtures) and any existing Design for Manufacture (DfM) constraints (such as draft angles, part extraction issues) that plague all established mass-production techniques, would allow the economic manufacture of a 
single unit, or ' $n=1$ ' as the engineers like to say. This simple but important principle had significant implications for product/engineering design because it means that there are no longer any geometric restrictions and forms can be readily achieved at almost any level of complexity. It also means that each object manufactured can be different from all the others, with little or no cost implications - the complete opposite of the constraints on conventional mass-production. The effects of this shift to being able to cost effectively produce single, bespoke products or components has prompted some significant work in the area of AM-based customisation and personalisation research.

The exact definitions or distinctions between customisation and personalisation have already received significant coverage (e.g. Sunikka and Bragge 2012) and a topic of enlightened discussion within this book. The majority of existing work using AM almost definitively falls within the sphere of personalisation and the creation of bespoke, one-off, person-specific, single user examples. However, more recent work has also explored the idea of 'mass-personalisation' and promoted the application of the technology for the production of mass-produced personalised products. Masspersonalisation raises some interesting research questions and challenges, most notably the requirement to engage masses of users who can personalise a product or an artefact. There are, however, issues surrounding the ownership of design rights and the attribution of liability (Moultrie and Bibb 2015). Design research supporting the concept (e.g. Franke et al 2010, Bernabei and Power 2013) are tackling these concerns, investigating the opportunities for 'Co-design' through direct end user engagement within the design process.

Indeed, the ongoing research and development within this field is growing and leads to an interesting question - is AM the future enabler of mass-personalisation? In order to address that particular question, it is important to document some of the previous, pivotal developments and applications of the technology within personalisation, which this chapter attempts to achieve. The examples presented here are by no means exhaustive but have been specifically selected by the author to help highlight both the scope and capability of AM technologies to deliver customised and 
personalised artefacts. To date, the majority of these examples lie with the Medical and Consumer application markets and it is here the impact of the technology for the creation of bespoke, one-off, person-specific artefacts can be truly demonstrated.

\section{Medical AM personalisation}

Some of the most well-known and celebrated examples of AM-based personalisation lie within the medical healthcare sector. Medical professionals, designers and engineers were some of the first to recognise the huge potential of AM techniques for the manufacture of personalised medical devices and implants. Since the late 1990's, they used the design freedom and flexibility of the manufacturing techniques to quickly and cost-effectively produce personalised geometry bespoke to the user. Interestingly, within medical applications, personalisation is perceived as vital to the functional performance of the object in question. Personalised medical product (or component) is often unseen or covert in their application and provides no self-expression by the user. There are numerous applications of AM within the medical healthcare sector, including dental implants / aligners, personalised implants and prosthetics, which are described below. However, one of the very first personalised, bestselling AM medical products which is probably the least well known is hearing aids (Figure 6.1).

\section{Personalised hearing aids}

Hearing aid manufactures have been engaged in the production of personalised products for many years. Much like finger prints, everyone's ear canal is unique, thus personalisation is a necessity. Prior to the adoption of AM, the manufacturing process often required several medical consultations and impressions of the inner ear to be taken for the production of moulds. This would be followed by a prolonged fabrication process to ensure correct fitting of final hearing aids and appropriate comfort to the user. While effective, the process was very time consuming and required numerous stages of fabrication with high levels of skill. With AM coming to light, this process was revolutionised dramatically, greatly reducing the time, skill and costs involved. However, the 
introduction of a digital manufacturing technique also requires the introduction and adoption of commentary and necessary technologies like 3D scanning and CAD, changing what was fundamentally a physical/analogue manufacturing process into a largely digital one. The stages of production can be summarised as follows:

- Customer/patient has a medical consultation where a wax impression of the inner ear is taken

- The wax impression is 3D scanned and converted into 3D CAD data of the hearing aid shell

- The 3D CAD data is then used to virtually construct the complete hearing aid by checking clearance for all necessary componentry

- The 3D CAD data of the shell is then 3D printed, cured and finished to the required level

- The 3D printed shell is then fitted with the necessary componentry and final assembly is completed.

INSERT Figure 6.1 Personlaised AM hearing aids-Courtesy of EnvisionTEC NEAR HERE

Hearing aids are a relatively simple but extremely effective application of AM and it may surprise most people that $99 \%$ of all hearing aids are now manufactured using some form of AM technology. It is also reported that up to $10,000,000$ pairs of AM hearing aids are being worn today by people all over the world and therefore this manufacturing process represents the largest implementation of personalisation ever recorded.

\section{Personalised orthodontic aligners}

Another highly successful implementation of AM-based personalisation are orthodontic aligners and specifically the work completed by Invisalign (Invisalign 2015). The system they developed involves the manufacture of a series (typically 13-52) of clear orthodontic aligners that eventually culminates in a straighten set of teeth for the patient (Figure 6.2). Usually, the process can take between six months and two years and normally requires a revised aligner to be worn every fortnight. The Invisalign system is supported by a very sophisticated 3D CAD platform that models the required 
movement of the teeth over the period of treatment until the final intended outcome is realised. Invisalign were very early adopters of Stereolithography (SL) technology and recognised its design freedom and flexibility that could be very successfully applied to manufacture all the subtly different aligners a patient would require over the course of their treatment. Unlike the example of hearing aids, where AM is specifically used to create the final outer shell, Invisalign use AM technology to create the intermediate tooling (mould) that allows the manufacture of the final aligners from thermo-formed clear thermoplastic. To date, Invisalign claim to have treated more than three million people which represents another hugely successful implementation of AM-based personalisation.

INSERT Figure 6.2 Invisalign orthodontic aligner NEAR HERE

\section{Personalised medical implants}

Other notable examples of personalisation within the medical field include those of implants and prostheses. Similar to hearing aids, the personalisation of these products existed long before the development and subsequent application of AM technologies. However, the level of personalisation was less sophisticated and the frequency of its implementation was on a much smaller scale. Prior to the introduction of AM, standardised medical implants would often be modified by the surgeons during the medical procedure itself to ensure an appropriate fit to the patient. The application of more complex or bespoke implants would often require a prolonged fabrication process prior to any surgery and then be subjected to a potentially lengthy medical validation process preceding any form of implantation. The introduction of AM techniques significantly reduced the cost and timescales involved in production of medical implants and also enabled a step-change in the achievable complexity and frequency of their use. A significant aspect of the research and development work within personalised AM implants has involved the introduction of biocompatible materials and approval by the various medical regulatory bodies such as the Medicines and Healthcare products Regulatory Agency (MHRA) within the United Kingdom and the Food and Drug 
Administration (FDA) within the United States of America. Titanium is a desirable build material for many applications due to its inert characteristics (such as corrosion resistance) and excellent mechanical properties. This led to its early development as a build material for metal-based AM technologies like Direct Metal Laser Sintering (DMLS) created by the EOS firm of Munich (Germany) and Selective Laser Melting (SLM) introduced at the Fraunhofer Institute ILT in Aachen in 1995. These technologies were used to manufacture direct parts for a variety of industries including aerospace, dental and medical. However, it was the biocompatibility of Titanium (non-toxic and acceptance by the body) and its capacity for osseointegration (a process which occurs when bone cells attach themselves directly to the titanium surface) that really elevated the use of AM for personalised orthopaedic implants. These highly desirable characteristic coupled with the design freedom of AM meant that extremely complex and bespoke orthopaedic implants can be generated that physically bond with bone and living tissue without any forms of additional adhesives. While personalised AM implants are being successfully used for dental, hip and knee procedures, it is within the field of cranio-maxillofacial surgery that AM has made some of the most significant impacts within personalisation.

The skull and jaw are arguably some of the most complex parts of the human skeletal system and the fabrication of implants to treat cranio-maxillofacial trauma and disease has historically been difficult. Through the utilisation of Magnetic Resonance Imaging (MRI) or Computed Tomography (CT) scanning, complex, personalised digital data of the patient can be captured and translated into useable 3D CAD format to facilitate the design of bespoke fitting implants. Notable work in the field has been completed by OBL - a manufacturer of implants for maxillofacial surgery, neurosurgery and plastic surgery (OBL 2015) who are part of the Materialise group (Materialise, 2015) as demonstrated in Figure 6.3.

INSERT Figure 6.3 Cranio-maxillofacial titanium implant (Materialise, 2015) NEAR HERE 
More recent innovations include the award winning work completed by Xilloc and LayerWise ( $\underline{\text { Xilloc }}$ 2015, LayerWise 2015) and their application of AM for the production of a complete lower titanium jaw implant (Figure 6.4). Developed in collaboration with the University of Hasselt BIOMED Research Institute (Belgium), LayerWise printed the complex Titanium mandible replacement that incorporated articulated joints and dedicated features. This was the first implementation and operation of its kind.

INSERT Figure 6.4 AM Titanium mandible replacement (Courtesy of Xilloc, the Netherlands) NEAR HERE

Personalised prosthetic fairings

Moving away from medical implants, but still within the medical/healthcare sector, AM has also been very successfully applied to manufacture of prosthetic fairings, specifically for leg amputees (Figure 6.5). Some of the most notable work to date has been completed by Bespoke Innovation, now a division of 3D Systems (3Dsystems 2015).

Functional or articulated prostheses have developed significantly in recent years and have the inherent capability to dramatically improve the quality of their user's life. While biomedical engineers have done an extraordinary job in delivering the functionality, these very intermit and personal devices have not received a similar level of industrial design. The application of AM for these products is not primarily functional, or indeed covert, but in fact purely aesthetic and explicit. The personalisation aspect of the work in this field is actually two-fold. It not only utilises personalised human body data for the generation of the fairing geometry, but also allows the personalisation of form and aesthetics for individualised expression. The process employed by Bespoke Innovations when working with the single leg amputees involves 3D Scanning of the 'soundside' or remaining leg which is used as reference geometry for the generation of the personalised fairing, ensuring appropriate body symmetry of the individual. The 3D body data is then applied as a template to construct a digital 3D model of the fairings panels with appropriate fixings to the 
underlying mechanical prostheses. The secondary personalisation aspect then involves the user selecting the aesthetic form and the final finish of the fairing. Here the process differentiates from all other medical-based applications of AM by encapsulating the end user's design aspirations.

INSERT Figure 6.5 Personalised prosthetic fairings by Bespoke Innovations NEAR HERE

The above overview of AM-enabled personalisation is not meant to be exhaustive, but merely provides key examples of its effective use within medical and healthcare sector. But it is fair to say, that personalisation of medical applications has not only been accepted as a recognised manufacturing solution, but also becomes more and more widespread as a means for individual aesthetic expression.

\section{Consumer AM personalisation}

Outside the medical and healthcare sector the existing examples of AM-based personalisation are much less numerous and many of them add little to the debate on the future of personalisation or increase the understanding of the opportunities that AM can provide. That being said, however, there are new developments of AM-based personalisation in such fields as footwear, lighting, ornaments, figurines and trinkets, which ignite a lot of interest in the current direction and applications of $A M$ within the consumer personalisation sector. The flexibility of AM along with other forms of digital manufacturing techniques are providing new possibilities for masspersonalisation, where every single product or component could be personalised to a single user's taste and requirements. Unlike 'Mass customisation' where the customisation of an object or system could be applicable to many potential users and based on a series of predetermined configurable options; mass-personalisation within the consumer sector seeks to involve the user within the design process. This could result in a potentially mass-produced, bespoke product for an individual user.

Personalised jewellery 
One of the earliest examples of AM enabled mass-personalisation was in the jewellery sector. Notably, the work of Future Factories - was amongst the first creative industries to employ AM to add value to their products through 'creative freedoms and manufacturing flexibility of digital technologies' (Future Factories 2015). The Icon pendant (2007) was a limited production of AM jewellery that utilised a 3D modelling script to continually transform the complex, organic form of the pendant over a specific time frame (Figure 6.6). Through a web-based portal, costumers could stop or freeze the transformation of the pendant and select to purchase a particular stage of this unique design metamorphosis. This approach not only used the design freedoms of AM to create a complex, organic form that would be highly impractical to reproduce with any other manufacturing techniques, it also embraced the flexibility of AM to provide a personalised product to ever single consumer. Furthermore, due to the 3D modelling script being used, every single design created within the limited production-run was instantly recognisable as being part of the Icon range, maintaining the product unique identity.

INSERT Figure 6.6 Future Factorise Icon pendant range NEAR HERE

This very early example of mass-personalisation using AM demonstrated the potential of the technology for consumer applications and also brought to light several key concepts that have been developed further within more recent examples of this growing trend. The main concept introduced was the generation of a 'seed object' or 'seed design'. This acts as the starting point for all subsequent transformations and specifically created to allow some form of personalisation by the intended end-user/customer. The second concept was the application of 'co-design' or 'co-creation' within the personalisation process, enabled through some form of web-based portal. The distinction between the activity of 'co-design' or 'co-creation' is quite narrow, but Sanders and Stappers (2008) define the difference between the two as: Co-design is a process that explicitly involves the end-user throughout the whole design process, whereas Co-creation is a boarder term that involves the enduser somewhere within the design process, irrespective of where it may occur. 
The growing trend for consumer-based, mass-personalisation using AM certainly seems to favour cocreation as the mechanism by which to involve the end-user. Every available example to date uses some form of seed object/design that has been initially created by a designer without any prior connection or communication with the end-user. The end-user is only presented with the opportunity to personalise the form or function of the seed object/design through a web-based portal prior to fabrication and therefore completing the co-creation process.

Several companies are now taking advantage of the flexibility that AM and other digital manufacturing techniques offer in order to provide opportunities for mass-personalisation through co-creation. Some of the current examples are extremely specific and concentrate solely on one product type, whereas others are more flexible and can accommodate a range of product variations.

\section{Personalised sex toys}

Possibly the most humours example of mass-personalisation through co-creation is the web-based portal 'Dildo Generator' (Dildo 2015) offering personalised sex toys. While somewhat whimsical, the system is actually quite well conceived and quickly allows anyone with almost no previous design skill or experience to design a personalised sex toy to suit their individual preferences. It is important to note that while AM is not directly used for the manufacture of the sex toy, it is used to create the mould tool for subsequent silicone moulding of bio-compatible materials. The dedicated system takes the end-user through a five stage creation process that not only allows the complete design but also the final purchasing of their personalised product.

Customers are first invited to select the type of sex toy they require from a predetermined list of product types (seed objects) before specifying certain size dimensions. The personalisation of the actual form is controlled by a simple and intuitive spline-based manipulation of the 2D profile by adding/subtracting spline points and simply dragging these around on the screen with a mouse. A 3D rendering of the design is updated in real time so the customers can instantly see the impact of their 
personalisation work (Figure 6.7). Once completed, the colour and surface texture can be specified before finally purchasing their unique personalised design.

\section{INSERT Figure 6.7 Dildo generator NEAR HERE}

While this example might be unusual and certainly has the potential to offend, it has been cited because it demonstrates a very important aspect within the growing trend of AM enabled masspersonalisation activities - the almost complete removal of any required design skill or expertise by the end-user/customer. As previously discussed within the introduction of this chapter, AM technologies offer unrivalled design freedom and any complexity of geometry can be created efficiently, repeatedly and economically. However, in order to achieve such complexity, a 3D modelling of a form in a CAD system is necessary. CAD is a fundamentally expert system requiring extensive skill and knowledge to operate. This fundamental prerequisite of the AM technology significantly limits the number of people that can effectively interact with and utilise its extraordinary capabilities to create personalised products. The development of simplified web-based portals that allow the real-time transformation of seed objects suddenly removes this barrier and enables a significantly larger population to interact with the technology - the scale consumer-based mass-personalisation has never experienced previously. However, not all of the current examples of web-based personalisation portals are quite so simple. With an increase in the product sophistication comes an obvious increase in the portal intricacy and complexity as described in the following section.

\section{Web-based portals for personalised consumer products}

Nervous System (Nervous, 2015) is a design studio which pioneered the use of generative design and AM techniques to provide a range of personalised products. Since 2007 they have developed a series of 'web-based generative design apps' that allow users to generate unique and affordable art, jewellery, and housewares manufactured using AM. Their web-based portal provides access to a range of dedicated software, including Cell Cycle (Figure 6.8) which offers a fairly extensive array of 
transformation options to modify and personalise a bespoke piece of AM jewellery featuring their distinct cellular architecture. Once again, the transformations are updated in real-time and the system allows the user to instantly see the results of their personalisation work on a rendering of the actual 3D data that will be used for manufacture. The user can specify the finish and material for their products, and is instantly informed about both the cost and delivery time of their design if purchased.

INSERT Figure 6.8 Nervous System - Cell Cycle software NEAR HERE

Possibly the most notable aspect of Nervous System's work involves the 'Kinematics Cloth' software which allows users to design a personalised 3D printed garment in real-time (Kinematics, 2015). The software is again accessible through their web-based portal and allows a variety of clothing items to be generated including dresses, skirts and shirts. Through a series of staged options, users can design the silhouette and hemline of their garment and determine the pattern of the garment's unique tessellated AM fabric structure. What makes this a particularly interesting example of personalisation is the ability for users to import their own 3D body scan data upon which to generate their personalised garments - allowing not only the form to be fully personalised but the garment to be custom-fitted to the user.

The examples above demonstrate the incredible potential for the personalisation of consumer products when using AM as the manufacturing solution. However, a distinct limitation of the portals developed so far is that of product variation. The vast majority tend to be dedicated to a single product type or product family (variations on a base product) and currently lack the versatility to allow the personalisation of a diverse range of merchandises. This is mainly linked to the complexity of developing a bespoke 3D modelling system that not only allows transformation of the 3D form but also accessibility through a web-based portal. These fundamental restrictions severely limit the wider opportunity for the personalisation of consumer products using co-creation and therefore number of examples currently available on the market. In order to achieve a greater opportunity for 
the personalisation of consumer products, a fundamental change in how product designs are created and made accessible through web-based portals is needed. One of the most developed and versatile mass-personalisation portals to date is the platform developed by Digital Forming (a UK based software company) and their proprietary ODO software (Digital Forming 2015). The rationale behind Digital Forming's work is to provide designers and businesses with a software solution and publishing platform to allow the generation of 'open' product designs being personalised by the user. The platform developed by Digital Forming aims to connect designers, retailers and online marketplaces with a network of digital manufacturers by offering a series of intuitive tools to exploit AM for mass-personalisation and on-demand production (Figure 6.9).

INSERT Figure 6.9 Digital Forming's platform NEAR HERE

While the number of example consumer products currently available through Digital Forming's platform is somewhat limited, the variation in product types is significant and presents the largest collection of personalised AM consumer products to date. However, what is more noteworthy is the potential of their platform and the likely impact it could have on the personalised consumer product market. The platform represents the first universal system to allow the wholesale creation of personalised consumer products and provides all the necessary tools for designers and businesses to fully engage within this developing market sector. This marks a distinct shift in the evolution of consumer product manufacturing, through embedding the opportunity for personalisation in almost any product design. As adoption increases, so will the number of personalised consumer products and it is anticipated to become far more commonplace in the next few years.

\section{Conclusion}

The examples discussed in this chapter are by no means complete and there are other instances where AM has been successfully utilised to provide opportunities for personalisation. Those cited, however, do demonstrate where AM is making a significant contribution to both the opportunity and application of personalisation. Within the medical healthcare sector AM has become an 
established and key manufacturing solution for the physical realisation of personalised products. It has not only allowed a step-change in the availability and suitability of products intimately personalised to the human form (implants), but also facilitated personalisation for individual expression (prosthetic fairings). The examples of hearing aids and orthodontic aligners reinforce its value as a manufacturing solution and demonstrate where AM has been vital in providing success for the mass-personalisation of commercial products.

Outside of the medical healthcare sector the examples of AM-based personalisation are less numerous and potentially more trivial in comparison. While there appears to be a real desire for the personalisation of consumer products, effective interaction with the technology and the generation of personalised 3D manufacturing data remains a significant barrier to further exploitation of the technology. The field of mass-personalisation requires continuous research and development work in order to provide the methodology and conceptual framework for the effective interaction with the technology. The ongoing development of web-based portals means that the ability to personalise a consumer product will soon become no more difficult than navigating a website. This apparent simplicity and availability will without doubts facilitate a steady increase in the future number of personalised consumer products available on the market.

So, returning to the original question - is AM the future enabler of mass-personalisation? AM is an incredible technology and provides extraordinary possibilities for design and personalisation. Within the medical healthcare sector it has already established itself as the key manufacturing solution for the mass-personalisation of commercial products. However, within consumer products sector it is currently being utilised 'on-demand' for low volume production while the means of engaging the consumer are fully developed. AM is slowly maturing as a manufacturing solution and continues to improve in terms of accuracy, speed and build materials. Will it become the future enabler of masspersonalisation of consumer products, you decide. 


\section{References}

Bernabei, R. \& Power, J., 2013. Designing together: end-user collaboration in designing a personalised product, 10th European Academy of Design Conference, 17-19 April 2013, Gothenburg, Sweden, pp. 1 - 12.

Burns, M. (1995), The freedom to create. Technology Management, 1995, 1(4), pp. 157-163.

Campbell, I. (1994) Design for rapid prototyping; developing a methodology. In Case, K and Newman, SE (ed) 10th National Conference on Manufacturing Research, Proceedings of the 10th National Conference on Manufacturing Research, Loughborough, UK, pp.521-525, ISBN: 0748402543.

Crump, S.S. (1992), Apparatus and method for creating three-dimensional objects, US Patent 5121329 A

Deckard, C.R. (1989), Method and apparatus for producing parts by selective sintering, US Patent 4863538 A

Franke, N., Kaiser, U. \& Schreier, M., 2010. The "I Designed It Myself" Effect in Mass Customization. Management Science, 56(1), pp. 125-140.

Hopkinson, N., and Dickens, P.M. 2003. Analysis of rapid manufacturing-using layer manufacturing processes for production. Proceedings of the Institute of Mechanical Engineers, Part C : Journal of Mechanical Engineering Science, 217 (C1), pp. 31-39

Hopkinson, N., Hague, R. J.M. and Dickens, P. M., eds. 2005. Rapid Manufacturing-An Industrial Revolution for the Digital Age, New York: John Wiley.

Hull, C.W. (1986), Apparatus for production of three-dimensional objects by Stereolithography, US Patent $4575330 \mathrm{~A}$

Makerspace (2015), http://www.makerspaceuk.com/, accessed April 2015

Moultrie, J. and Bibb, R. (2015), Design for Additive Manufacturing (D4AM). EPSRC Grant reference $\mathrm{EP} / \mathrm{N005953/1}$ 
Sanders, E. \& Stappers, P.J. (2008c) Co-creation and the new landscapes of design. CoDesign, 4(1), pp. 5-18.

Sunikka, A. \& Bragge, J., 2012. Applying text-mining to personalization and customization research literature - Who, what and where? Expert Systems With Applications, 39(11), pp.10049-10058.

\section{Websites:}

3DSystems (2015), www.3dsystems.com, accessed June 2015

Digital Forming, 2015, www.digitalforming.com, accessed August 2015

Dildo (2015), www.dildo-generator.com, accessed June 2015

Future factorise (2015), www.futurefactories.com, accessed June 2015

Invisalign (2015), http://www.invisalign.co.uk, accessed May 2015

Kinematics (2015), www.n-e-r-v-o-u-s.com/kinematicsCloth, accessed July 2015

LayerWise (2015), http://www.layerwise.com/, accessed June 2015

Materialise (2015), http://cranio-maxillofacial.materialise.com/, accessed May 2015

Nervous (2015), www.n-e-r-v-o-u-s.com, accessed July 2015

OBL (2015), http://www.oblparis.com/?lang=en, accessed May 2015.

Xilloc (2015), http://www.xilloc.com/patients/stories/total-mandibular-implant/, accessed May $\underline{2015}$. 